\title{
An approach to optimize the settings of actuated signals
}

\author{
Xinguo JIANG ${ }^{1 *}$, Yanjun QIU², Sheng RUAN ${ }^{3}$ \\ 1. School of Transportation and Logistics, Southwest Jiaotong University, Chengdu 610031, China \\ 2. School of Civil Engineering, Southwest Jiaotong University, Chengdu 610031, China \\ 3. Zhejiang Provincial Hongtu Transportation Construction Co., Ltd., 2031 Jiangling Road, Hangzhou 310003, China
}

\begin{abstract}
Traffic-actuated signal employs relatively complex control logic to regulate traffic flow. Introduction of control variables into the traffic-actuated system contributes to system operational flexibility and complexity, and also complicates the system with uncertainties. The paper proposes two tentative methods to optimize the actuated signal parameters: basic requirements of controller parameters and analytical model, and macroscopic computer simulation. It is concluded that when the actuated signal operates within the volume/capacity range of 0.4 to 0.6 , it will create the most significant benefits; the research suggests that minimum green time in the main street shall be set long enough to meet the required demand, preferably at the $60 \%$ of the main street capacity. In order to ensure less control delay in a semi-actuated intersection, relatively small values of vehicle extension (e.g., $2.5 \mathrm{~s}$ ) and maximum green time are recommended to be assigned to the less important street.
\end{abstract}

Key words: actuated signals; optimization; parameter settings; computer simulation

(C) 2011 JMT. All rights reserved.

\section{Introduction}

$\mathrm{T}$ raffic-actuated signal employs relatively complex control logic to regulate traffic flow. It provides the control system with a significant flexibility, but also leads the control system to a complicated situation. Compared to the pre-timed signal system, actuated signal system can be responsive to serve the timedependent dynamic traffic demand, aiming at reducing the system total delay, queued vehicles and then improving the system performance. Introduction of control variables into the traffic-actuated system contributes to system operational flexibility and complexity. As a downside, complicated nature of traffic actuated signal operation brings about a lot of uncertainties and unexpected results into the system, so that practical engineers and signal designers find it difficult to make full utilization of the potential benefits of actuated signal. The complexity stems from the actuated controller settings, time-dependent traffic demand pattern, and the interaction between motorized traffic and non-motorized traffic. The operational performance of isolated actuated intersections or a set of coordinated actuated intersections largely depends on traffic patterns and actuated control-

Received Feb. 25, 2011; revision accepted Mar. 8, 2011

*Corresponding author. E-mail: jiangxi1@msu.edu doi: 10.3969/j.issn.2095-087X.2011.01.010 ler parameters [1]. Especially when considering applying the actuated signals in the progression applications [2], inappropriate signal settings can result in excessive delays and disruption of traffic progression on major movements. A well-designed actuated control parameter that responds appropriately to traffic demand will have substantial influences on system's measures of effectiveness (MOE), such as system-wide total delay, fuel consumption, queued vehicles, stop and travel time. The purpose of the paper is to develop different analytical methods to achieve approximately optimal controller settings for the purpose of minimizing the signal intersection delay. The methods are further investigated through the macroscopic computer simulation to determine whether they can be applied in a practical sense to estimate the delay of vehicle-actuated signals.

\section{Literature review}

Traffic-actuated control signal has been used since the early 1930s. During the past 80 years, a number of researchers have developed different theories focusing on optimizing the controller settings, detector placement, and the relationship between them, using various traffic simulation software and analytical models. The relationship between delay and controller setting parameters for a simple vehicle actuated type was originally studied by Morris and Pak-Poy [3]. They found that associated with each traffic flow condition there was an optimal vehicle 
interval for which the average delay per vehicle was Riniminiced and tha $325733^{3}$ value of the optimal vehicle interval decreased and became more critical as the traffic flow increased. Darroch [4] proposed a method to obtain optimal estimates of the unit extension, which minimized total vehicle delays.

A lot of disagreements and discussions arise from the discrepancy of computer simulation models. Lin [5] stated that NETSIM model [6] was not accurate enough to account for the probability of premature phase termination caused by the failure of queuing vehicles to extend green time, under the assumption that every vehicle in a queue could extend a green duration until the queue dissipated completely. The author recommended the utilization of RAPID model for determination of controller setting. RAPID model was a microscopic simulation model capable of duplicating the dynamic and probabilistic interactions between queuing vehicles and present detector [7]. Bullen et al. [8] developed EVIPAS, which was a computer model for the optimal design of a vehicle-actuated traffic signal. EVIPAS was designed to analyze and optimize a wide range of intersection, phasing, and controller characteristics of an isolated, fully actuated traffic signal. The model could provide optimal timing settings for pre-timed, semi-actuated, fully actuated or volume-density control using a variety of measures of effectiveness chosen by users. Courage and Luh [9] developed guidelines for determining the trafficactuated signal control parameters, which would produce the optimal operation identified by SOAP84. They also evaluated the existing signal control parameters on an individual traffic-actuated signal. Bullen [10] used the EVIPAS simulation and optimization model to analyze traffic-actuated signals. The results showed that vehicle extension should be more than $4 \mathrm{~s}$ regardless of detector location and approach speed, which somewhat contradicted to previous research results.

In terms of the analytical model, three researches ([5], [11-12]) made significant contribution to determine the optimization of actuated controller settings. Courage and Papapanou [11], Lin [5], and Mazdeyasna [13] proposed an extension to Webster's delay model to explicitly deal with certain operation parameter setting. The modified version of Webster's formula [14] included one unique feature that two coefficients of sensitivity were considered to account for level of sensitivity of the average delay to the ratio of effective green to cycle length, and degree of sensitivity of average delay to saturation ratio. Akcelik [12] developed a theoretical model to optimize actuated controller settings by means of estimating exponential headway distribution, although a lot of further work was needed to validate and calibrate those formulas. One technical report from FHWA [2] used a particular approach to set controller parameters based on the timings from signal optimization programs: producing algorithms and procedures to translate the fixed time settings output from the optimization programs into equivalent settings for actuated controllers in a system.

In summary, substantial efforts have been invested in the optimization of actuated signal controller variables, although some research results and analytical models remain in the theoretical stage and real-life and simulation data are needed to calibrate and validate those theories. Due to the characteristics of uncertainty and the complexity of actuated signal operation, no permanent controller settings can be developed to meet all kind of situations. Courage [15] stated that the most successful cases of locally actuated intersection control were based on intuitive mechanical models, which were primitive and defied purely analytical treatment. Therefore, the contribution of this paper is expected to serve as an extension to work that has been previously achieved.

\section{Theoretical development}

Basically, for actuated signal controllers there are three important operating parameters, which can be referred to as "controller setting", because they must be physically set in the controller with switches, keypads, or some other electrical means. Before the implementation of actuated controller, those controller parameters should be carefully determined, tested and optimized, which will exert significant influences on the operation of the intersection. Theoretical development to optimize controller parameters encompasses two components: basic controller parameters and analytical model.

\subsection{Basic parameters}

The paper tries to find out the basic requirements for each controller setting from the rudimentary definition, the calculation formula, and the inherent relationships. There are three principal types of actuated signal controllers: semi-actuated, fully actuated, and volumedensity. They have different mechanisms and operation logics and are suitable for different roadway and traffic conditions and traffic control schemes. The difference in choice of controller settings attributes to the operational discrepancy. Table 1 lists the different variables used in each type of actuated controllers.

Volume-density control was designed primarily for junctions where the phases frequently run to maximum and where there is heavy traffic in one phase at one time of the day and in a different phase at another time of day. The controller settings will be automatically adaptive to fluctuations in flow at each phase, which adds to the dif- 
Table 1 Control settings in different actuated signals DOI: $10.1007 / \mathrm{BF} 03325733$

\begin{tabular}{ll}
\hline $\begin{array}{l}\text { Actuated } \\
\text { signal type }\end{array}$ & Controller settings \\
\hline Semi-actuated & $\begin{array}{l}\text { Minimum green time at major and } \\
\text { minor street, maximum green time at } \\
\text { minor street, and unit extension }\end{array}$ \\
Fully-actuated & $\begin{array}{l}\text { Minimum green time and maximum } \\
\text { green time at both streets, } \\
\text { and unit extension }\end{array}$ \\
Volume-density & $\begin{array}{l}\text { Minimum green time at both streets, } \\
\text { time-dependent unit extension, } \\
\text { gap reduction rate, time before reduction, } \\
\text { time to reduce and minimum gap }\end{array}$ \\
\hline
\end{tabular}

ficulties of effectiveness evaluation and actual operation complexity. Therefore, the paper chooses individual fully actuated signals as the research entity and focuses on three main parameters: minimum green time, maximum green time, and unit extension (vehicle interval, or passage time). The similar theory can be extended to the semi-actuated signal.

\section{(1) Minimum green time.}

Minimum green time refers to the shortest time that should be provided for a green interval during a traffic phase. It is equal to the sum of the initial portion and the unit extension. The basic function of setting minimum green time is to serve vehicles queuing from last cycle and ensure enough time to pass through the intersection during next signal period. In the definition of minimum green time, initial portion can be defined as the first portion of the green phase that an actuated controller has timed out, for vehicles waiting between the detector and the stop line during the red phase. The calculation formula can be expressed as follows:

$$
\begin{aligned}
& M n G=I P+U, \\
& I P=\left(h n+K_{1}\right), \\
& U=\frac{X+W+L}{1.47 u}, \\
& M n G=\left(h n+K_{1}\right)+\frac{X+W+L}{1.47 u},
\end{aligned}
$$

where, $M n G$ stands for minimum green time; $I P$ is initial portion; $U$ is unit extension; $u$ is the average speed, in $\mathrm{mph} ; X$ is the distance between detector and stop line, in $\mathrm{ft}$; $W$ is the width of the cross street, in $\mathrm{ft} ; L$ is the length of the vehicle, in $\mathrm{ft}$; $h$ is the average headway, in $\mathrm{s}$, and the suitable value is $2 \mathrm{~s} ; n$ is the number of vehicles per lane waiting between detectors and the stop line; and $K_{1}$ is the starting delay, suitable value, $3.5 \mathrm{~s}$.
Most of the variables in Eq.(4) can be calibrated and validated from the field survey and empirical experiences. One important parameter that should be mentioned is the detector location. Suggested distance for the detection location, such as in Manual of Uniform Traffic Control Devices, has been determined from observations or experiences; but when the random traffic arrival pattern and traffic demand are considered, it is not optimal enough to account for all factors. Morris and Pak-Poy [3] pointed out that the location of simple vehicle actuated and more complex controller detector should be considered together with unit extension to achieve optimal control settings. Consequently, the critical relationship between minimum green time and unit extension can be translated into detector location and unit extension.

\section{(2) Maximum green time.}

Maximum green time refers to the longest time that can be provided for a green interval during a traffic phase. It is equal to sum of minimum green time and extension limit, usually 1.25 to 1.50 times of computed green intervals. Maximum green limits the maximum green duration allowable to a signal phase after a vehicle actuates a detector of competing phase. In terms of traffic operation conditions, reasonable determination of maximum green time is essential to the whole intersection delay. Based on the functionality and logic of actuated signal, it is expected that under low to moderate volumes (volume/capacity ratio, $v / c$, from 0.44 to 0.72 ) actuated signal will be automatically responsive to traffic variation. When traffic volumes increase substantially and reach significant level $(v / c$ ratio higher than 0.75 ), actuated signal will be operated under its maximum time settings during a specific time period. Under the saturation condition, the operation of a vehicleactuated signal is not different from a pre-timed signal because the signal is continuously operated under the maximum settings. So, in order to calculate optimal maximum green time at a designated intersection, an actuated signal is designed as a pre-timed intersection under extreme condition. This is not an arbitrary statement; some researchers ([3] [5], [11], and [16]) have made the similar comments about setting maximum green time.

\section{(3) Unit extension.}

Unit extension is the minimum time by which a green phase can be increased during the extendable portion after an actuation on the phase, which determines the longest duration in which detectors can be left unoccupied without prompting the termination of a green duration. If such effective vehicle intervals are short, both queuing vehicles and vehicles not in a queue may have great difficulties extending a green phase. So, from this 
aspect traffic demand requires that a vehicle-extension

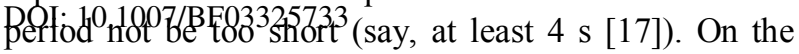
other hand, long effective vehicle intervals may allow vehicles separated by long headways to extend a green phase, but significant queuing length will form at competing approaches, which in turn may worsen the level of service.

\subsection{Analytical model}

In this section, the fully actuated intersection will be discussed. The mechanism of how the green time is extended will be carefully explored under different situations. The main idea of optimizing the maximum green time is to calculate the average green time, which is the average value of maximum green time under each case multiplied with each individual probability. According to the functionality of fully-actuation intersection, the green time is allocated into two cases: the minimum green time and minimum green time plus effective unit extension.

Most traffic constitutes two types of vehicles: the first is the platoon vehicles which closely follow the preceding vehicle; the second is the free-moving vehicles that travel without interacting with the vehicle ahead. Therefore, the dichotomized headway distribution will be employed in this study.

The probability for headway $h>t$ is

$$
P(h>t)=1-\alpha \mathrm{e}^{-t / \bar{h}_{\mathrm{f}}}+(1-\alpha) \mathrm{e}^{-\left(t-t_{\mathrm{m}}\right) /\left(\bar{h}_{\mathrm{b}}-t_{\mathrm{m}}\right)},
$$

where, $\bar{h}_{\mathrm{f}}$ is the average headway for free-moving vehicles, which can be determined under the circumstance of free traffic flow where the vehicles travel at the desired/optimal speed; $\bar{h}_{\mathrm{b}}$ is the average headway for bunched or constrained vehicles, which can be directly observed from the field; $t_{\mathrm{m}}$ is the shift in the curve, considered as the minimum headway (depending on the saturation flow rate); and $\alpha$ is the percent of free vehicles (not in the platoons), which can be justified through a field data survey.

Case $(a)$

For case (a), the phase occupying right-of-way does not receive extended green time, based on the condition that time headway is larger than one unit extension, which means during the last one unit-extension time period of the minimum green time $\left(G_{a}\right)$, no actuation call is caused by arriving traffic in the right-of-way phase. The probability for this case $\left(P_{a}\right)$ :

$$
\begin{aligned}
& P_{a}=P(h>U)=1-\alpha \mathrm{e}^{-U / \bar{h}_{\mathrm{f}}}+(1-\alpha) \mathrm{e}^{-\left(U-t_{\mathrm{m}}\right) /\left(\bar{h}_{\mathrm{b}}-t_{\mathrm{m}}\right)}, \\
& G_{a}=G_{\min } .
\end{aligned}
$$

Case (b)

The rest of conditions will be case $(b)$ that time headway space is less than one unit extension, $h \leqslant U$. Once the headway between two adjacent vehicles is smaller than unit extension, an effective actuation call will be immediately activated by the arriving platoon traffic, and thus unit extension will be extended until any the headway is larger than the unit extension. Therefore, the probability $\left(P_{b}\right)$ that headway less than unit extension can be computed with the following formula:

$$
\begin{gathered}
P_{b}=P(h \leq U)=[1-P(h \geq U)]= \\
\alpha \mathrm{e}^{-U / \bar{h}_{\mathrm{f}}}-(1-\alpha) \mathrm{e}^{-\left(U-t_{\mathrm{m}}\right) /\left(\bar{h}_{\mathrm{b}}-t_{\mathrm{m}}\right)} .
\end{gathered}
$$

The following section will focus on how to calculate the average extended green time. The problem is that some consecutive unit extensions overlap together, causing some extendable green time to be wasted. Fig. 1 graphically illustrates how the actuated phase works in either semi- or fully-actuated intersection.

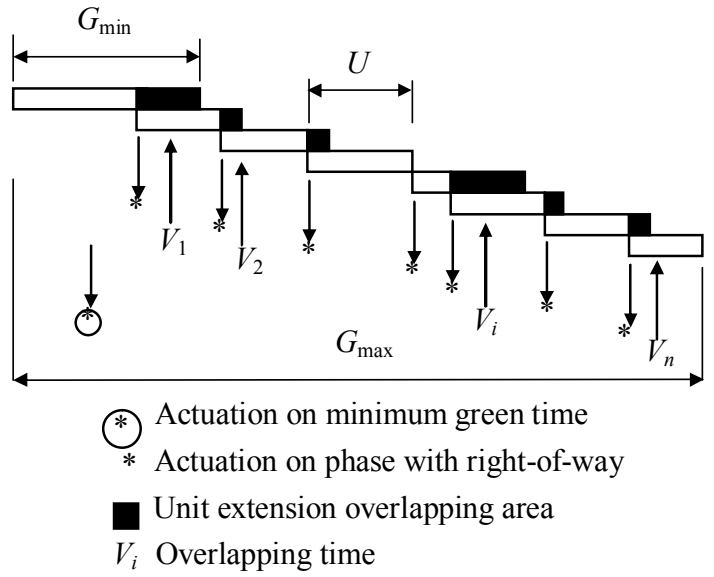

Fig. 1 Actuated signal traffic actuation mechanism

Based on the information from Fig. 1, we have the following formula:

$$
\begin{gathered}
V_{\mathrm{e}}=\left(U-V_{1}\right)+\left(U-V_{2}\right)+\ldots+ \\
\left(U-V_{i}\right)+\ldots+\left(U-V_{n}\right)= \\
n U-\sum_{i}^{n} V_{i}=n(U-\bar{V}),
\end{gathered}
$$

where, $V_{\mathrm{e}}$ is the effective extended green time extension; $V_{i}$ is unit extension overlapping for unit extension $i ; \bar{V}$ is the average overlapping unit extension; and $n$ is the number of vehicle arriving at $V_{\mathrm{e}}$. So, effective unit extension multiplying traffic arrival rate equals to the number of vehicles arriving during time $V_{\mathrm{e}}$.

$$
\begin{aligned}
& n(U-\bar{V}) \lambda=n, \\
& \bar{V}=U-\frac{1}{\lambda},
\end{aligned}
$$




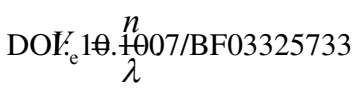

As for the number of vehicles arriving during the effective extended green time extension, we can estimate the value by using observation data from the field. So,

$$
G_{b}=G_{\min }+V_{\mathrm{e}} \text {. }
$$

Therefore, the average green time for one approach in a fully actuated intersection can be expressed as:

$$
G=P_{a} G_{\min }+P_{b}\left(G_{\min }+V_{\mathrm{e}}\right),
$$

where, $\lambda$ is the traffic arrival rate for the calculating phase; $G_{\min }$ is the minimum green time for the calculating phase; and $U$ is the unit extension.

One might consider that optimal maximum green time is approximately equal to the minimum green time plus the average green time, $G$. Based on this assumption, we might be able to calculate the optimal or nearoptimal maximum green time from the mathematical perspective.

\section{Computer simulation}

With the increasing interest on actuated signal, more and more actuated signal simulation programs have been developed and used for the study of this kind. As indicated in the literature interview, a lot of commercial software is available, such as RAPID, VIPAS, EVIPAS, SOAP, SYNCHRO, CORSIM, and VISSIM. Simulation modeling has become an extremely important approach to analyze complex actuated signal system. The introduction of computer simulation in actuated signal parameters will help to establish the relationship between different parameters from a practical standpoint. According to the availability and applicability of software, the macroscopic simulation program SYNCHRO is utilized in the study to optimize the control parameters. The semi-actuated intersection is chosen as an example.

The objectives of the simulation study are two-folded: (1) the effect of the different combinations of $v / c$ ratios of the main street and side street, together with vehicle extension on intersection control delay; and (2) the effect of maximum green time of the side street combined with unit extension on intersection control delay.

The main benefit of deployment of actuated signal is that signal timing will be responsive to the traffic random arrival and time-dependent traffic demand. There is an assumption under this statement. The randomness of traffic arrivals and demand fluctuations will fall within the ability of actuated signal timing adjustment range. Excessive demand will cause the actuated signals to virtually operate as fixed-time signals. Different volume to capacity ratios of main street volume will be expected to significantly affect the intersection control delay. An isolated uncoordinated actuated intersection is modeled by SYNCRHO. Fig. 2 demonstrates the variation of uncoordinated actuated intersection control delay under $v / c$ ratios of the side street and the main street in the range of 0.2 to 1.2 , and $2 \mathrm{~s}$ unit extension.

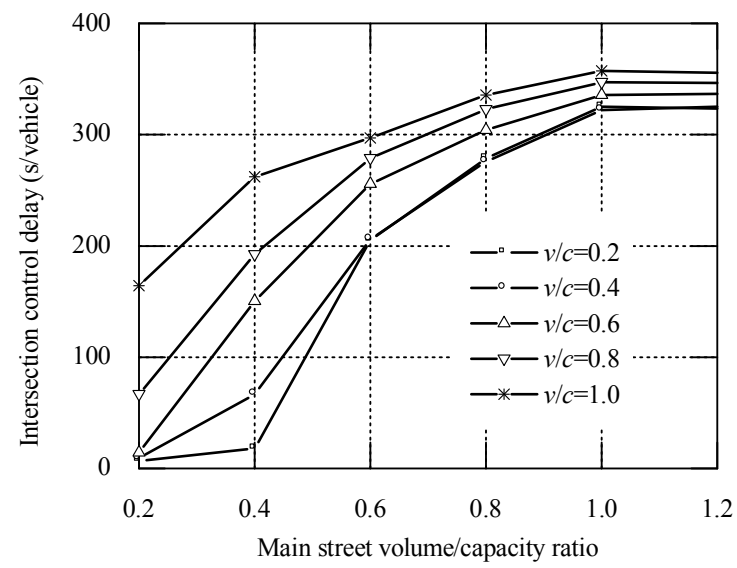

Fig. 2 Relationship between main street $v / c$ ratio and intersection delay $(U=2 \mathrm{~s})$

Fig. 2 also shows that when main street $v / c$ ratio reaches 0.6 , the intersection control delay gradually becomes insensitive to the increasing $v / c$ ratios. This phenomenon is also applicable to different $v / c$ ratios in the side street, that is, for each scenario the curve pattern and shape are similar. Examination of a series of vehicle extension cases identifies that intersection control delay is significantly sensitive to the $v / c$ ratio in the main street $v / c$ ratio falling within 0.4 to 0.6 , regardless of $v / c$ ratios of the side street. This is an important observation that when actuated signal operating with the $v / c$ range from 0.4 to 0.6 is expected to create significant benefits, which consequently suggests that minimum green time in the main street shall be long enough to meet the required demand, specifically, preferably at the $60 \%$ main street capacity.

Table 2 illustrates that unit extension becomes insen-

Table 2 Control delay and unit extension related to main street $v / c$ ratio (side street $v / c=0.2$ )

\begin{tabular}{cccccc}
\hline & \multicolumn{5}{c}{ main street $v / c$ ratios } \\
\cline { 2 - 6 } & 0.2 & 0.4 & 0.6 & 0.8 & 1 \\
\hline 2.0 & 6.6 & 9.0 & 14.1 & 66.8 & 164.4 \\
2.5 & 6.8 & 9.3 & 14.2 & 66.8 & 164.4 \\
3.0 & 7 & 9.5 & 14.3 & 66.8 & 164.4 \\
3.5 & 7.3 & 9.7 & 14.5 & 66.8 & 164.4 \\
4.0 & 7.5 & 9.9 & 14.5 & 66.8 & 164.4 \\
\hline
\end{tabular}


sitive to control delay with the increasing main street $v / c$

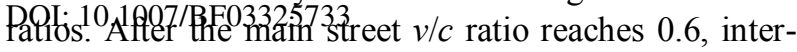
section control delay almost remains the same regardless of the increasing unit extension, which also confirms the previous findings.

Further study is conducted to examine the relationship between the side street maximum green time and the intersection control delay under both nearly congested and low to moderate conditions. The different curves in Fig. 3 stand for different unit extension values. For the case (main street traffic flow 2000 vehicle/h and side street traffic flow 500 vehicle/h), the beginning segment of the curves follows the same pattern, due to the limited availability of the green time. The curves reach the summit when the maximum green time is set to $30 \mathrm{~s}$. However even the fluctuation exists in the curves, the absolute oscillation is not substantial. The conclusion drawn from the study is that the best value of the maximum duration does not vary much with respect to varying vehicle extensions and the results of the fixed-timing scheme may reasonably be used as a guide to the value of the maximum period.

It is observed from Fig. 4 that a set of curves have the

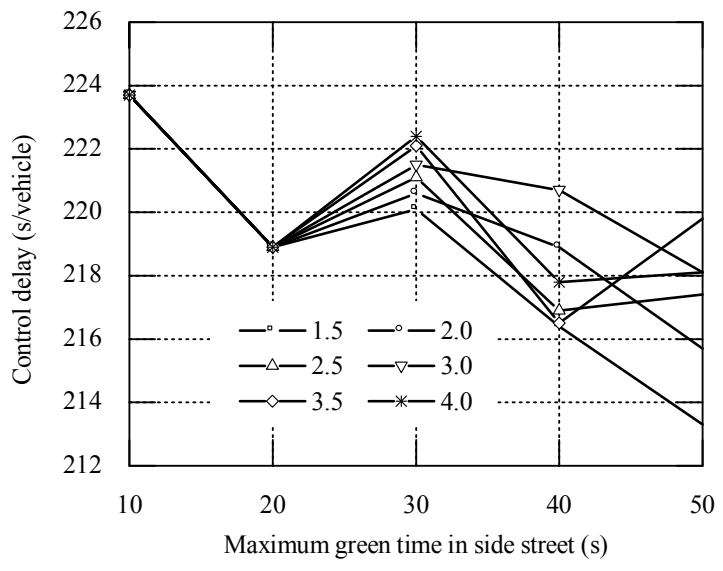

Fig. 3 Control delay and maximum green time of the side street (nearly congested)

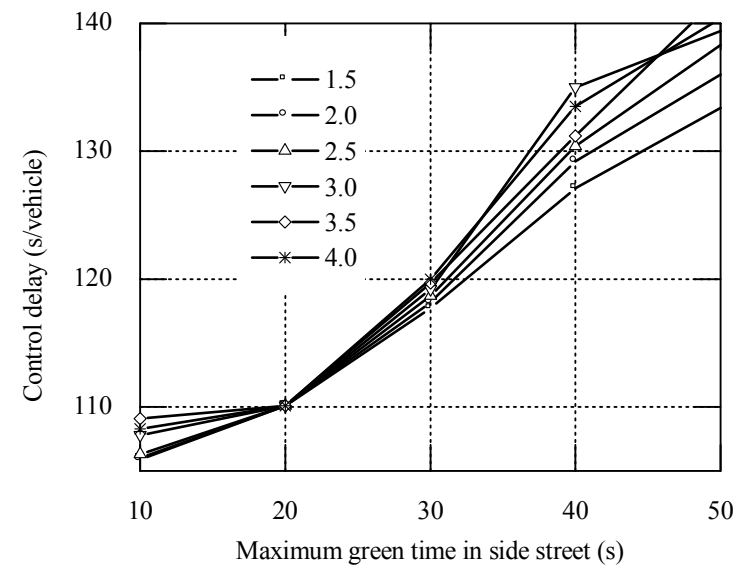

Fig. 4 Control delay and maximum green time of the side street (low to moderate) same trend pattern, that is, control delay increases with the maximum green time.

The total intersection control delay will increase with the cycle length under under-saturated traffic conditions. At the same time, the figure also demonstrates that for the same maximum green time the smaller vehicle extension the less control delay is. The increasing gapping out times in the side street will spare more green time to the main street and will serve more traffic. Under the premise of ensuring a required level of service for the side street, in order to minimize the whole intersection control delay relatively small values of vehicle extension and maximum green time are recommended for the side street.

\section{Conclusion}

In the paper, two tentative methods have been developed to optimize the actuated signal parameters: basic requirements of controller parameters and analytical model, and macroscopic computer simulation. Through analyzing the basic requirements, the paper tries to explain the conceptual requirements of each parameter from the perspectives of basic definitions and calculation formulas. From the analytical model, micromechanism of fully-actuated intersection is explored to reveal the inherent relationships between minimum green time and maximum green time, assuming that the traffic arrival headway follows a dichotomized distribution. Through the macroscopic computer simulation, the effects of the combination of $v / c$ ratio of the main street and the side street, together with vehicle extension on intersection control delay is discussed in details, and the effect of maximum green time of the side street combined with unit extension on intersection control delay is studied as well. It is concluded that when the actuated signal operates within the $v / c$ range of 0.4 to 0.6 , it will create the most significant benefits; the research suggests that minimum green time in the main street shall be set long enough to meet the required demand, preferably at the $60 \%$ of the main street capacity. In order to ensure less control delay in a semi-actuated intersection, relatively small values of vehicle extension (e.g., $2.5 \mathrm{~s}$ ) and maximum green time are recommended to be assigned to the less important street.

\section{Acknowledgements}

The research is supported by the Fundamental Research Funds for the Central Universities (SWJTU09CX042) and National Natural Science Foundation of China (NSFC-50978222). 


\section{References 300325733}

[1] Transportation Research Center at University of Florida, Capacity analysis of traffic-actuated intersections: Final Report, NCHRP PROJECT 3-48: 1996.

[2] A. Skabardonis, Progression through a series of intersections with traffic actuated controllers, Report DTFH6187-R-00027, Vol. 1, Technical Report, FHWA. U.S. Department of Transportation, Oct. 1988.

[3] R.W.T. Morris, P.G. Pak-Poy. Intersection control by vehicle actuated signals, Traffic Engineering and Control, 1967(10): 288-293.

[4] J.N. Darroch, G.F. Newell, R.W.J. Morris, Queues for a vehicle-actuated traffic light, Operational Research, 1964, 12(6): 882-895.

[5] F.B. Lin, Optimal timing setting and detector lengths of presence mode full-actuated control. Transportation Research Record, 1985(1010): 37-45.

[6] FHWA, U.S., Traffic network analysis with NETSIM: a user guide. Report FHWA-IP-80-3. Department of Transportation, January 1980.

[7] F.B. Lin, Optimal timing setting and detector lengths of presence mode full-actuated control, Draft Final Report. Office of University Research, U.S. Department of Transportation, December 1984.

[8] A.G.R Bullen, N. Hummon, T. Bryer, et al., EVIPAS: A computer model for the optimal design of a vehicleactuated traffic signal, Transportation Research Record, 1987(1114): 103-110.

[9] K.G. Courage, J.Z. Luh, Development of guidelines for implementing computerized timing design at traffic actuated signals, Final Report Vol. 1, Isolated Intersection Implementation, Transportation Research Center, University of Florida, February 1989.

[10] A.G.R. Bullen, The effects of actuated signal settings and detector placement on vehicle delay, Transportation Research Record, 1989(68).

[11] K.G. Courage, P.P. Papapanou, Estimation of delay at traffic-actuated signals. Transportation Research Record, 1977(630): 17-20.

[12] R. Akcelik, Estimation of green times and cycle time for vehicle-actuated signals. In: Transportation Research Record, 1994(1457): 63-72.

[13] F.B. Lin, M. Farrokh, Delay models of traffic-actuated signal controls. Transportation Research Recor, 1987(905): 33-38.

[14] F.V. Webster, Traffic signal settings. In: Transportation Research circular 212, TRB, National Research Council, Washington, D.C., January 1980.

[15] K.G. Courage, Delay models of traffic-actuated signal controls, In: F.B. Lin, M. Farrokh. In: Transportation Research Record, 1987(905): 33-38.

[16] National Research Council, Special Report 209: Highway capacity manual. TRB, Washington, D.C., 1985.

[17] F.V. Webster, B.M. Cobbe, Traffic signals, Road Research Technical Paper No.56, London, Her Majesty's Stationery Office, 1966.

(Editor: Dongju CHEN) 